\title{
Extending quantum control of time-independent systems to time-dependent systems
}

\author{
Zhen-Yu Wang and Ren-Bao Liv* \\ Department of Physics, The Chinese University of Hong Hong, Shatin, N. T., Hong Kong, China
}

\begin{abstract}
We establish that if a scheme can control a time-independent system arbitrarily coupled to a generic finite bath over a short period of time $T$ with control precision $O\left(T^{N+1}\right)$, it can also realize the control with the same order of precision on smoothly time-dependent systems. This result extends the validity of various universal dynamical control schemes to arbitrary analytically time-dependent systems.
\end{abstract}

PACS numbers: 03.67.Pp, 03.65.Yz

\section{INTRODUCTION}

Quantum systems interact with their environments (or baths). This results in errors in controlling evolution of a quantum system, such as decoherence and unwanted dynamics. Inspired by phase-refocusing techniques in magnetic resonance spectroscopy [1, 2], various schemes of quantum dynamical control [3-22] have been developed in the context of quantum information processing to average out undesired coupling through fast open-loop modulation on the system evolution. These dynamical control schemes have advantages of correcting errors without measurement, feedback, or redundant encoding [6]. The simplest one is dynamical decoupling (DD) [3-15], which aims at preservation of arbitrary states (i.e., quantum memory or NULL quantum control) by achieving a trivial identity evolution. Recent experiments [23-28] have demonstrated the performance of DD. More general aims are to implement non-trivial quantum evolutions [16-22]. Arbitrarily accurate dynamical control can be achieved using a concatenated design [19].

A quantum dynamical control is called universal if it has errors up to an order in short evolution time $T$ for an arbitrary finite bath. Most universal schemes [6-14, 17-19] are designed for time-independent systems and their applicability to time-dependent systems is unclear, except an explicit extension [29] of Uhrig dynamical decoupling (UDD) [10, 30] to analytically time-dependent systems.

In this paper, we prove a theorem that if a dynamical control has errors to the $N$ th order in short evolution time $T$ for a generic time-independent system, it will automatically achieve the same order of precision for analytically timedependent systems. The theorem establishes the validity of universal DD in Refs. [6-14], optimized pulses in Ref. [17], and dynamical quantum error correction [18, 19] on nonequilibrium baths. In addition, it greatly simplifies designing new universal dynamical control schemes since we just need to work with time-independent models.

We present the proof in Sec. $\coprod$ and draw the conclusion in Sec.III

*Electronic address: rbliu@cuhk.edu.hk

\section{CONTROL OF TIME-DEPENDENT SYSTEMS}

\section{A. Universal control of time-independent systems}

Let us first consider a target system coupled to a bath through a time-independent Hamiltonian

$$
H_{S B}=\sum_{\alpha=0}^{D-1} S_{\alpha} \otimes B_{\alpha},
$$

where $S_{\alpha}$ and $B_{\alpha}$ are operators of the system and bath, respectively, and in particular, $S_{0} \equiv I_{S}$ is the identity operator and $B_{0}$ is the bath internal interaction. We assume that $S_{\alpha}$ and $B_{\alpha}$ are bounded in spectrum so that a perturbative expansion of the system-bath propagator driven by $H_{S B}$ converges for a short evolution time $T$. Otherwise, the coupling is generic, that is, the details of $B_{\alpha}$ are unspecified. In Eq. (1), $\mathcal{S} \equiv\left\{S_{\alpha} \mid \alpha=0, \ldots, D-1\right\}$ does not have to be the basis of the full Lie algebra. For example, in the pure dephasing Hamiltonian of a qubit coupled to a bath, $\mathcal{S}=\left\{I, \sigma_{z}\right\}$, which only generates a sub-algebra of a qubit.

Control of the system is implemented by applying a Hamiltonian $V_{c, T}(t)$ on the system. To realize a desired system evolution $Q$ (e.g., a quantum gate) over a given duration of time $T, V_{c, T}(t)$ scales with $T$ so that $(\hbar=1)$

$$
\begin{aligned}
U_{c, T}(t) & \equiv \mathcal{T} \exp \left[-i \int_{0}^{t} V_{c, T}(\tau) d \tau\right] \\
& =\mathcal{T} \exp \left[-i \int_{0}^{t / T} V_{c}(\theta) d \theta\right] \equiv U_{c}(t / T),
\end{aligned}
$$

where $\mathcal{T}$ denotes the time-ordering operator, and $V_{c}(\theta)=$ $T V_{c, T}(T \theta)$. We consider the case of perfect control, that is, $U_{c, T}(T)=U_{c}(1)=Q$ is the desired control of the system. Under influence of the environment, the system-bath propagator reads

$$
U(T)=\mathcal{T} \exp \left(-i \int_{0}^{T}\left[H_{S B}+V_{c, T}(t)\right] d t\right) .
$$

The errors induced by $H_{S B}$ can be isolated in the interaction picture by writing $U(T)=Q U_{E}(T)$, where the error propagator

$$
\begin{aligned}
U_{E}(T) & \equiv \mathcal{T} \exp \left[-i \int_{0}^{T} U_{c, T}^{\dagger}(t) H_{S B} U_{c, T}(t) d t\right] \\
& =\mathcal{T} \exp \left[-i T \int_{0}^{1} U_{c}^{\dagger}(\theta) H_{S B} U_{c}(\theta) d \theta\right] .
\end{aligned}
$$


We suppose that the control $V_{c, T}(t)$ has been designed to suppress the errors due to $H_{S B}$ up to the $N$ th order of the evolution time $T$, which is assumed short, that is,

$$
U_{E}(T)=U_{\Omega}\left[1+O\left(T^{N+1}\right)\right]
$$

where $U_{\Omega}$ is an operator commuting with a certain set of system operators $\Omega$. A control $V_{c, T}(t)$ is universal if Eq. (5) holds for arbitrary time-independent $B_{\alpha}$. Ref. [19] shows that $V_{c, T}(t)$ can be designed to achieve Eq. (5) with arbitrary $N$ and $Q \neq I_{S}$ for a general time-independent model [Eq. (1)]. For the special case of dynamical decoupling, $Q=I_{S}$ and all the operators in $\Omega$ are preserved; if $\Omega$ spans the full algebra of the system, $U_{\Omega}$ is a pure bath operator and any system states (hence quantum correlations) will be protected [14].

\section{B. Generalization to time-dependent systems}

A time-dependent version of Eq. (1) reads

$$
H_{S B}^{\prime}(t)=\sum_{\alpha=0}^{D-1} S_{\alpha} \otimes B_{\alpha}^{\prime}(t)
$$

where the generic bath operators are assumed analytic in time:

$$
B_{\alpha}^{\prime}(t)=\sum_{p=0}^{\infty} B_{p}^{\prime(\alpha)} \frac{1}{p !} t^{p}
$$

We also assume that the bath operators $B_{p}^{\prime(\alpha)}$ are bounded in spectrum. We are to prove the following theorem.

Theorem. If $V_{c, T}(t)$ realizes Eq. (5) for an arbitrary timeindependent Hamiltonian in Eq. (1), it will realize the control with the same order of precision for an arbitrary timedependent Hamiltonian in Eq. (6), that is, the system-bath propagator commutes with the system operator set $\Omega$ up to an error $O\left(T^{N+1}\right)$,

$$
\begin{aligned}
U^{\prime}(T) & \equiv \mathcal{T} \exp \left(-i \int_{0}^{T}\left[H_{S B}^{\prime}(t)+V_{c, T}(t)\right] d t\right) \\
& \equiv Q U_{E}^{\prime}(T)=Q U_{\Omega}^{\prime}\left[1+O\left(T^{N+1}\right)\right],
\end{aligned}
$$

where $U_{\Omega}^{\prime}$ commutes with the operator set $\Omega$.

Proof. We write

$$
U_{E}(T)=e^{-i B_{0} T} \tilde{U}_{E}(T)
$$

and

$$
\begin{gathered}
\tilde{U}_{E}(T)=\mathcal{T} \exp \left[-i T \int_{0}^{1} \sum_{\alpha=1}^{D-1} U_{c}^{\dagger}(\theta) B_{\alpha}(\theta) U_{c}(\theta) d \theta\right], \\
B_{\alpha}(\theta)=e^{i B_{0} T \theta} B_{\alpha} e^{-i B_{0} T \theta}=\sum_{k=0}^{\infty}\left[i B_{0}, B_{\alpha}\right]_{k} \frac{(T \theta)^{k}}{k !}
\end{gathered}
$$

with $\left[i B_{0}, B_{\alpha}\right]_{k+1} \equiv\left[i B_{0},\left[i B_{0}, B_{\alpha}\right]_{k}\right]$ and $\left[i B_{0}, B_{\alpha}\right]_{0} \equiv B_{\alpha}$. The perturbative expansion of $\tilde{U}_{E}(T)$ in short time $T$ reads

$$
\tilde{U}_{E}(T)=1+\sum_{n=1}^{\infty} \sum_{\vec{\alpha}=1}^{D-1} \sum_{\vec{p}=0}^{\infty} T^{n+|\vec{p}|} S_{n}^{\vec{\alpha}, \vec{p}} \otimes \mathcal{B}_{n}^{\vec{\alpha}, \vec{p}},
$$

with short-hand notations $\sum_{\vec{\alpha}=1}^{D-1} \equiv \sum_{\alpha_{1}=1}^{D-1} \cdots \sum_{\alpha_{n}=1}^{D-1}, \sum_{\vec{p}=0}^{\infty} \equiv$ $\sum_{p_{1}=0}^{\infty} \cdots \sum_{p_{n}=0}^{\infty}$, and $|\vec{p}| \equiv \sum_{j=1}^{n} p_{j}$, where the bath and system operators are,

$$
\begin{aligned}
\mathcal{B}_{n}^{\vec{\alpha}, \vec{p}} & \equiv \frac{\left[i B_{0}, B_{\alpha_{1}}\right]_{p_{1}}}{p_{1} !} \cdots \frac{\left[i B_{0}, B_{\alpha_{n}}\right]_{p_{n}}}{p_{n} !} \\
S_{n}^{\vec{\alpha}, \vec{p}} & \equiv \int_{0}^{1} U_{c}^{\dagger}\left(\theta_{1}\right) S_{\alpha_{1}} U_{c}\left(\theta_{1}\right) \theta_{1}^{p_{1}} \int_{0}^{\theta_{1}} U_{c}^{\dagger}\left(\theta_{2}\right) S_{\alpha_{2}} U_{c}\left(\theta_{2}\right) \theta_{2}^{p_{2}} \\
& \times \cdots \int_{0}^{\theta_{n-1}} U_{c}^{\dagger}\left(\theta_{n}\right) S_{\alpha_{n}} U_{c}\left(\theta_{n}\right) \theta_{n}^{p_{n}} d \theta_{1} d \theta_{2} \cdots d \theta_{n}
\end{aligned}
$$

respectively.

For the time-dependent Hamiltonian $H_{S B}^{\prime}(t)$, the expansion of $\tilde{U}_{E}^{\prime}(T) \equiv\left(\mathcal{T} e^{-i \int_{0}^{T} B_{0}^{\prime}(t) d t}\right)^{\dagger} U_{E}^{\prime}(T)$ has a similar form

$$
\tilde{U}_{E}^{\prime}(T)=1+\sum_{n=1}^{\infty} \sum_{\vec{\alpha}=1}^{D-1} \sum_{\vec{p}=0}^{\infty} T^{n+|\vec{p}|} S_{n}^{\vec{\alpha}, \vec{p}} \otimes \mathcal{B}_{n}^{\prime \vec{\alpha}, \vec{p}},
$$

where $\mathcal{B}_{n}^{\prime \vec{\alpha}, \vec{p}} \equiv\left(B_{p_{1}, 0}^{\prime\left(\alpha_{1}\right)} / p_{1} !\right) \cdots\left(B_{p_{n}, 0}^{\prime\left(\alpha_{n}\right)} / p_{n} !\right)$ with $B_{p, 0}^{(\alpha)}$ defined by the expansion of $B_{\alpha}^{\prime}(t)$ in the interaction picture, $\left(\mathcal{T} e^{-i \int_{0}^{t} B_{0}^{\prime}(s) d s}\right)^{\dagger} B_{\alpha}^{\prime}(t)\left(\mathcal{T} e^{-i \int_{0}^{t} B_{0}^{\prime}(s) d s}\right) \equiv \sum_{p=0}^{\infty} B_{p, 0}^{(\alpha)} t^{p} / p !$.

By assumption, $V_{c, T}(t)$ realizes Eq. (5) for a generic timeindependent $H_{S B}$. In Appendix, we give an explicit construction of $\left\{B_{\alpha}\right\}$, for which the set of bath operators $\left\{\mathcal{B}_{n}^{\vec{\alpha}, \vec{p}}|n+| \vec{p} \mid \leq\right.$ $N\}$ is linear independent. Eqs. (5) and (9) indicate that $\tilde{U}_{E}(T)$ commutes with $\Omega$ up to the $N$ th order in $T$. If the bath operators $\left\{\mathcal{B}_{n}^{\vec{\alpha}, \vec{p}}|n+| \vec{p} \mid \leq N\right\}$ in Eq. (12) are linear independent (nonzero, of course), $S_{n}^{\vec{\alpha}, \vec{p}}$ must commute with the system operator set $\Omega$ for $n+|\vec{p}| \leq N$. Since Eq. 15] is also an expansion of $S_{n}^{\vec{\alpha}, \vec{p}}, \tilde{U}_{E}^{\prime}(T)$ and hence $U_{E}^{\prime}(T)$ commute with the system operator set $\Omega$ up to an error $O\left(T^{N+1}\right)$.

The theorem extends the validity of universal dynamical control to analytically time-dependent systems. Note that in Eq. (1), $\mathcal{S}=\left\{S_{\alpha} \mid \alpha=0, \ldots, D-1\right\}$ does not have to span the full algebra of the system but should contain the identity operator $I_{S}$. Therefore the theorem does not rely on the specific algebra generated by $\mathcal{S}$ and is general for any system-bath interactions provided that the total generic Hamiltonian includes a free bath term $I_{S} \otimes B_{0}$. It should be stressed that because the details of the bath operators $B_{\alpha}^{\prime}(t)$ are unspecified, the dynamical control is still valid if we do the following variation:

$$
H_{S B}^{\prime}(t) \rightarrow H_{S}(t) \otimes I_{B}+\sum_{\alpha=0}^{D-1} S_{\alpha} \otimes B_{\alpha}^{\prime}(t),
$$

where $H_{S}(t)$ is a bounded system operator in the space spanned by $\mathcal{S}$ and analytic in time and $I_{B}$ is the identity operator of the bath. Actually, the first term in the right-hand 
side of Eq. (16) can be readily absorbed into the system-bath coupling. The drift errors introduced by the system's internal Hamiltonian $H_{S}(t)$ are also eliminated.

The operator $H_{S B}^{\prime}(t)$ is required to be bounded for any $t \in$ $[0, T]$. However, to implement a system evolution $U_{c, T}(T)=$ $U_{c}(1)=Q \neq I_{S}$, the control $V_{c, T}(t)$ must scale as $\sim 1 / T$; this scaling induces a faster evolution on the system as $T \rightarrow 0$ in the limit of instantaneous pulses and this is the requirement of any dynamical control schemes to suppress errors: the evolution of the system driven by the control field $V_{c, T}(t)$ needs to be faster than the bath evolution induced by $H_{S B}$ or $H_{S B}^{\prime}(t)$.

\section{CONCLUSIONS}

We have proved that a universal dynamical control which implements a quantum evolution of a system up to an error $O\left(T^{N+1}\right)$ in total evolution time $T$ for a generic timeindependent system-bath Hamiltonian automatically suppresses errors to $O\left(T^{N+1}\right)$ for any analytically time-dependent Hamiltonians. The extension of various universal dynamical control schemes [6- 14, 17-19] to arbitrary analytically timedependent systems is therefore established. This result also simplifies the design of other universal control schemes. The current research raises an interesting question for future study: Are there minimal models for which a control scheme works with a certain degree of precision will work with the same order of precision for arbitrary systems, time-independent or not?

\section{Acknowledgments}

This work was supported by Hong Kong GRF CUHK402209, the CUHK Focused Investments Scheme, and National Natural Science Foundation of China Project 11028510 .

\section{Appendix}

Here we give an explicit construction of $\left\{B_{\alpha}\right\}$, so that the set of bath operators $\left\{\mathcal{B}_{n}^{\vec{\alpha}, \vec{p}}|n+| \vec{p} \mid \leq N\right\}$ in Eq. (12) are linear independent. For that purpose we prove the following lemma.

Lemma. For a finite number $R$, there exists a construction of $K^{\prime}$ Hermitian operators $\left\{O_{k}\right\}_{k=1}^{K^{\prime}}$, such that all the operator products $O_{k_{1}} O_{k_{2}} \cdots O_{k_{r_{j}}}$ for different sequences $\left(k_{1} k_{2} \cdots k_{r_{j}}\right)$ with $1 \leq k_{l} \leq K^{\prime}$ and $1 \leq r_{j} \leq R$ are linear independent.

Proof. Let $K=K^{\prime}+1$ and $\left\{|l\rangle \mid l=0, \ldots,\left(K^{R}+1\right) K\right\}$ be an orthonormal basis. We construct the following Hermitian operators in the Hilbert space expanded by this basis

$$
O_{k}=\sum_{l=0}^{K^{R}}|l\rangle\langle K l+k|+\text { h.c.. }
$$

Examination of the projection $|0\rangle\langle 0| O_{k_{1}} O_{k_{2}} \cdots O_{k_{r_{j}}}$ shows that the operator $O_{k_{1}} O_{k_{2}} \cdots O_{k_{r_{j}}}$ contains one and only one component of the form $|0\rangle\langle l|$, explicitly, $|0\rangle\left\langle\left(k_{1} k_{2} \cdots k_{r_{j}}\right)_{K}\right|$, where

$$
\left(k_{1} k_{2} \cdots k_{r_{j}}\right)_{K} \equiv K^{r_{j}-1} k_{1}+\cdots+K k_{r_{j}-1}+k_{r_{j}},
$$

is a number of base $K$ with $1 \leq k_{l} \leq K-1$. Therefore all the operator products $O_{k_{1}} O_{k_{2}} \cdots O_{k_{r_{j}}}$ for different sequences $\left(k_{1} k_{2} \cdots k_{r_{j}}\right)$ with $1 \leq k_{l} \leq K^{\prime}$ and $1 \leq r_{j} \leq R$ are linear independent.

An explicit construction of $\left\{B_{\alpha}\right\}$ reads

$$
\begin{aligned}
& B_{\alpha}=\sum_{r=0}^{N-1}|r\rangle\langle r| \otimes h_{r}^{(\alpha)}, \text { for } \alpha \geq 1, \\
& B_{0}=\sum_{r=0}^{N-1}|r\rangle\langle r+1| \otimes I_{h}+\text { h.c. }
\end{aligned}
$$

where $\{|r\rangle \mid r=0, \ldots, N-1\}$ is an $N$-dimensional orthonormal basis with the periodic condition $|r+N\rangle=|r\rangle, h_{r}^{(\alpha)}$ is an Hermitian operator, and $I_{h}$ is the identity operator. Using the Lemma, we have a construction of the operators $\left\{h_{r}^{(\alpha)} \mid 0 \leq\right.$ $r \leq N-1,1 \leq \alpha \leq D-1\}$ such that all operator products $h_{r_{1}}^{\left(\alpha_{1}\right)} \cdots h_{r_{n}}^{\left(\alpha_{n}\right)}$ are linear independent for different sequences of $\left(\alpha_{1}, \ldots, \alpha_{n}\right)$ and $\left(r_{1}, \ldots, r_{n}\right)$. We decompose $B_{0}=B_{+}+B_{-}$ with $B_{+} \equiv \sum_{r=0}^{N-1}|r\rangle\langle r+1| \otimes I_{h}$ and $B_{-}=\left(B_{+}\right)^{\dagger}$. Some calculation gives

$$
\frac{\left[i B_{+}, B_{\alpha}\right]_{p}}{p !}=\sum_{r=0}^{N-1}|r\rangle\langle r+p| \otimes\left(\sum_{k=0}^{p} c_{p}^{(k)} h_{r+p-k}^{(\alpha)}\right),
$$

where $p \geq 0$ and $c_{p}^{(k)}=(-1)^{k} i^{p} /[k !(p-k) !]$ is a non-zero coefficient. Therefore

$$
\begin{aligned}
& \left\langle 0\left|\mathcal{B}_{n}^{\vec{\alpha}, \vec{p}}\right||\vec{p}|\right\rangle=\left\langle 0\left|\frac{\left[i B_{+}, B_{\alpha_{1}}\right]_{p_{1}}}{p_{1} !} \cdots \frac{\left[i B_{+}, B_{\alpha_{n}}\right]_{p_{n}}}{p_{n} !}\right||\vec{p}|\right\rangle \\
& =\left(\sum_{k=0}^{p_{1}} c_{p_{1}}^{(k)} h_{p_{1}-k}^{\left(\alpha_{1}\right)}\right)\left(\sum_{k=0}^{p_{2}} c_{p_{2}}^{(k)} h_{p_{1}+p_{2}-k}^{\left(\alpha_{2}\right)}\right) \cdots\left(\sum_{k=0}^{p_{n}} c_{p_{n}}^{(k)} h_{p_{1}+\cdots+p_{n}-k}^{\left(\alpha_{n}\right)}\right) .
\end{aligned}
$$

There is one and only one operator product $h_{p_{1}}^{\left(\alpha_{1}\right)} h_{p_{1}+p_{2}}^{\left(\alpha_{2}\right)} \cdots h_{p_{1}+\cdots+p_{n}}^{\left(\alpha_{n}\right)}$ in $\left\langle 0\left|\mathcal{B}_{n}^{\vec{\alpha}, \vec{p}}\right||\vec{p}|\right\rangle$. If $n+|\vec{p}| \leq N$, the operators $h_{p_{1}}^{\left(\alpha_{1}\right)} h_{p_{1}+p_{2}}^{\left(\alpha_{2}\right)} \cdots h_{p_{1}+\cdots+p_{n}}^{\left(\alpha_{n}\right)}$ are linear independent for different $\{n, \vec{\alpha}, \vec{p}\}$ according to the Lemma. Thus for $n+|\vec{p}| \leq N, n \geq 1$, and $|\vec{p}| \geq 0,\left\langle 0\left|\mathcal{B}_{n}^{\vec{\alpha}, \vec{p}}\right||\vec{p}|\right\rangle$ and hence $\mathcal{B}_{n}^{\vec{\alpha}, \vec{p}}$ are linear independent for different $\{n, \vec{\alpha}, \vec{p}\}$. 
[1] M. Mehring, Principles of High Resolution NMR in Solids (Spinger-Verleg, Berlin, 1983), 2nd ed.

[2] A. Schweiger and G. Jeschke, Principles of Pulse Electron Paramagnetic Resonance (Oxford Unversity Press, New York, 2001).

[3] L. Viola and S. Lloyd, Phys. Rev. A 58, 2733 (1998).

[4] M. Ban, J. Mod. Opt. 45, 2315 (1998).

[5] P. Zanardi, Phys. Lett. A 258, 77 (1999).

[6] L. Viola, E. Knill, and S. Lloyd, Phys. Rev. Lett. 82, 2417 (1999).

[7] L. Viola and E. Knill, Phys. Rev. Lett. 90, 037901 (2003).

[8] P. Wocjan, Phys. Rev. A 73, 062317 (2006).

[9] K. Khodjasteh and D. A. Lidar, Phys. Rev. Lett. 95, 180501 (2005).

[10] G. S. Uhrig, Phys. Rev. Lett. 98, 100504 (2007).

[11] G. S. Uhrig, Phys. Rev. Lett. 102, 120502 (2009).

[12] G. S. Uhrig and S. Pasini, New J. Phys. 12, 045001 (2010).

[13] J. R. West, B. H. Fong, and D. A. Lidar, Phys. Rev. Lett. 104, 130501 (2010).

[14] Z.-Y. Wang and R.-B. Liu (2010), arXiv:1006.1601v2 (Phys. Rev. A, in press).

[15] W. Yang, Z.-Y. Wang, and R.-B. Liu, Front. Phys. 6, 2 (2011).

[16] L. Viola, S. Lloyd, and E. Knill, Phys. Rev. Lett. 83, 4888 (1999).

[17] S. Pasini, P. Karbach, C. Raas, and G. S. Uhrig, Phys. Rev. A 80, 022328 (2009).
[18] K. Khodjasteh and L. Viola, Phys. Rev. Lett. 102, 080501 (2009).

[19] K. Khodjasteh, D. A. Lidar, and L. Viola, Phys. Rev. Lett. 104, 090501 (2010).

[20] J. Clausen, G. Bensky, and G. Kurizki, Phys. Rev. Lett. 104, 040401 (2010).

[21] G. Bensky, E. Brion, F. Carlier, V. M. Akulin, and G. Kurizki, Europhys. Lett. 89, 10011 (2010).

[22] J. R. West, D. A. Lidar, B. H. Fong, and M. F. Gyure, Phys. Rev. Lett. 105, 230503 (2010).

[23] M. J. Biercuk, H. Uys, A. P. VanDevender, N. Shiga, W. M. Itano, and J. J. Bollinger, Nature 458, 996 (2009).

[24] J. F. Du, X. Rong, N. Zhao, Y. Wang, J. H. Yang, and R. B. Liu, Nature 461, 1265 (2009).

[25] E. R. Jenista, A. M. Stokes, R. T. Branca, and W. S. Warren, J. Chem. Phys. 131, 204510 (2009).

[26] G. de Lange, Z. H. Wang, D. Ristè, V. V. Dobrovitski, and R. Hanson, Science 330, 60 (2010).

[27] C. A. Ryan, J. S. Hodges, and D. G. Cory, Phys. Rev. Lett. 105, 200402 (2010).

[28] G. A. Álvarez, A. Ajoy, X. Peng, and D. Suter, Phys. Rev. A 82, 042306 (2010).

[29] S. Pasini and G. S. Uhrig, J. Phys. A: Math. Theor. 43, 132001 (2010).

[30] W. Yang and R.-B. Liu, Phys. Rev. Lett. 101, 180403 (2008). 